\title{
Risk Stratification for Athletes and Adventurers in High-Altitude Environments: Recommendations for Preparticipation Evaluation
}

Aaron D. Campbell, MD, MHS; Scott E. McIntosh, MD, MPH; Andy Nyberg, MD; Amy P. Powell, MD; Robert B. Schoene, MD; Peter Hackett, MD

From Family and Sports Medicine, University of Utah Health Care, Salt Lake City, Utah (Dr Campbell); the Division of Emergency Medicine, University of Utah Health Care, Salt Lake City, Utah (Drs McIntosh and Nyberg); the Department of Orthopedics, University of Utah, Salt Lake City, Utah (Dr Powell); Bay Area Pulmonary/Critical Care Medical Associates, Berkeley/Oakland, California (Dr Schoene); and the Institute for Altitude Medicine, Telluride, Colorado (Dr Hackett).

\begin{abstract}
High-altitude athletes and adventurers face a number of environmental and medical risks. Clinicians often advise participants or guiding agencies before or during these experiences. Preparticipation evaluation (PPE) has the potential to reduce risk of high-altitude illnesses in athletes and adventurers. Specific conditions susceptible to high-altitude exacerbation also important to evaluate include cardiovascular and lung diseases. Recommendations by which to counsel individuals before participation in altitude sports and adventures are few and of limited focus. We reviewed the literature, collected expert opinion, and augmented principles of a traditional sport PPE to accommodate the highaltitude wilderness athlete/adventurer. We present our findings with specific recommendations on risk stratification during a PPE for the high-altitude athlete/adventurer.
\end{abstract}

Key words: preparticipation evaluation, altitude, chronic disease AND altitude, wilderness sports, wilderness athlete

\section{Introduction}

High-altitude trekking and mountaineering are popular internationally. Davies et $\mathrm{al}^{1}$ suggest 30000 trekkers annually attempt Mt. Kilimanjaro. Records from sagarmatha National Park, Solukhumbu, Nepal, report 37000 Everest trekkers annually as of April 2015. ${ }^{2}$ Hackett and Roach $^{3}$ estimate 30 million Western States visitors, 1200 McKinley climbers, 10000 Rain climbers, and over 4000 Aconcagua climbers are at risk for altitude illness each year. These authors go on to say "Increasingly, physicians are confronted with questions of prevention and treatment of high-altitude medical problems as well as the effects of altitude on preexisting medical conditions."

Few physicians are both qualified and available to provide advice or pretrip counsel to such individuals.

The authors report no conflicts of interest.

This article appears in a "Care of the Wilderness and Adventure Athlete" special issue, jointly published by Clinical Journal of Sport Medicine and Wilderness \& Environmental Medicine.

Corresponding author: Aaron D. Campbell, MD, MHS (e-mail: aaroncampbell100@gmail.com).
Guidelines on preparing individuals for altitude adventures largely do not exist beyond expert opinion from a select few articles. Doan and Luks ${ }^{4}$ provide an excellent resource for sports and travel at altitude in individuals with asthma. Collective recommendations reflecting other conditions, however, are rare or nonexistent. Here, we offer suggestions for the health provider who is not an expert in high-altitude medicine on how to facilitate a preparticipation evaluation (PPE) directed to the high-altitude athlete or adventurer. The authors of this article do this by presenting a strategy for risk stratification based on guidelines for the prevention and treatment of acute altitude illness and also consider the presence of health conditions of individuals that pose risk for participation in high-altitude adventures in addition to known risks of injury and illness at altitude.

Giving "clearance" may not be the role of the screening provider regardless of their level of training. Wilderness sports are not typically governed by rules and regulations in the same way as traditional or organized sports. Although high-altitude-guided trips in many cases require a physician clearance, this process is not 
standardized nor outlined for the screening provider. With a focused outline on areas specific to altitude adventures, the screening provider can assess risk by evaluating the health status and stability of chronic conditions of the participant. The provider can then risk stratify the high-altitude athlete/adventurer and educate him/her based on those findings.

Building on principles of a traditional $\mathrm{PPE},{ }^{5}$ an evaluation for altitude adventures should emphasize altitude-specific issues. In general, this will involve screening for life-threatening/disabling conditions and for conditions that can predispose to injury or illness. Secondary objectives should determine overall health and facilitate a discussion on health-related topics. ${ }^{5}$

Ultimately, the individual's health status will predict the extent of the PPE, and the history is the most important component. ${ }^{5}$ Physical examinations may not always occur, as in the case of clinicians reviewing health history forms of clients on guided expeditions or providing counsel to individuals over the phone or Internet. Referral to specialists may be indicated for any issues needing further evaluation. Concerns for heart disease such as hypertrophic cardiomyopathy, long QT syndrome, and Wolff-Parkinson-White will likely indicate a cardiology referral, as would complicated pulmonary or gastrointestinal concerns need specialty evaluation. We recommend being thorough yet reasonable to avoid unnecessary studies or interventions.

Because medical care is not always immediately available on high-altitude adventures, the other members of the group may become the health care providers. Because of this, patients with chronic medical conditions have an obligation to inform their teammates of the severity, extent, and treatment options for their conditions. We encourage self-awareness in the high-altitude athlete/adventurer and open disclosures to a team about the status of health conditions.

\section{Methods}

Using the databases of MEDLINE, Cochrane, and Embase, we cross-referenced the key term "preparticipation evaluation" with variations of "altitude," "chronic disease AND altitude," "wilderness sports," and "wilderness athlete" to identify articles published in English from peer-reviewed journals. We also referenced book sections to facilitate discussions on altitude physiology.

\section{Basic Principles of Altitude Physiology}

Understanding adaptation to high altitude is essential to provide guidelines that optimize both the health and success of the high-altitude athlete/adventurer training or performing at high altitude. High altitude is defined as
1500 to $3500 \mathrm{~m}(\sim 4950-11500 \mathrm{ft})$, very high altitude as 3500 to $5500 \mathrm{~m}(\sim 11500-18050 \mathrm{ft})$, and extreme altitude as $>5500 \mathrm{~m}(\sim 18050 \mathrm{ft})$, over which longterm acclimatization is considered impossible. ${ }^{3}$

The body can acclimatize to achieve near sea level values of exercise performance while training at modest altitudes (approximately $2500 \mathrm{~m} / 8200 \mathrm{ft}$ ). But, even prolonged duration and training at moderate to very high altitudes does not bring exercise ability back to sea level performance. ${ }^{6}$ Variability to acclimatization, likely based on genetics, results in a broad bell-shaped curve of human physiologic responses to altitude. Some individuals never fully adapt to altitude and can incur acute, subacute, and/ or chronic altitude illness. The first public awareness of the effect of high altitude on athletic competition occurred in the 1968 Olympics in Mexico City $(2200 \mathrm{~m} / 7200 \mathrm{ft})$ where the best athletes in the world from lower altitudes experienced poorer performances in aerobic events than had been expected.

Aerobic capacity decreases on ascent to high altitude because of the decrease in partial pressure of oxygen and thus availability of oxygen in the inspired air. ${ }^{6,8-10}$ The fraction of oxygen in the earth's atmosphere remains constant at 0.2093 , whereas the density of the air as measured by the barometric pressure $(\mathrm{Pb}, \mathrm{mm} \mathrm{Hg})$ decreases. ${ }^{11}$ To obtain the same amount of oxygen requires moving increasingly greater volumes of air in the lungs, so ventilation increases on ascent to high altitude. . $^{12,13}$ It occurs immediately and results in a higher alveolar $\mathrm{PO}_{2}$ and lower alveolar $\mathrm{PCO}_{2}$, thus supplying a higher level of oxygen for gas exchange than would otherwise be expected if ventilation did not increase. The subsequent progressive increase in alveolar ventilation over days and weeks increases alveolar and arterial $\mathrm{PO}_{2}$.

Further acclimatization occurs from 2 adaptations: (1) the compensatory excretion of bicarbonate from the kidneys leading to a metabolic acidosis (partial compensation of the respiratory alkalosis) and (2) increasing sensitization of the carotid body. Responses vary widely, which has implications for adaptation, performance, and susceptibility to altitude illnesses (HAI), whereas at low altitude, the manifestations of these characteristics go unnoticed.

Dyspnea is experienced on the first ascent, even at modest altitudes. This dyspnea decreases somewhat on acclimatization to a specific altitude while ventilatory drive and resting and exercise ventilation are increasing.

Gas exchange across the alveoli occurs normally, but the lower alveolar $\mathrm{PO}_{2}$ imposes a diffusion limitation of oxygen from the air to the blood resulting in further hypoxemia. ${ }^{14,15}$ As high altitude increases, the diffusion limitation for oxygen increases such that there is not sufficient time for full equilibration of alveolar $\mathrm{PO}_{2}$ with capillary $\mathrm{PO}_{2}$. This process is accentuated by exercise when the transit time of 
blood across the pulmonary capillary decreases, resulting in more arterial desaturation.

Arterial desaturation can become extreme at very high altitudes, thus limiting oxygen delivery to the tissues and further decreasing exercise capacity. Gas exchange is improved with hypoxic pulmonary vasoconstriction with better matching of ventilation and perfusion relationships and an increase in cardiac output. These processes occur very quickly after exposure to a hypoxic environment. However, hypoxic pulmonary vasoconstriction can lead to high-altitude pulmonary edema (HAPE) in susceptible individuals.

To augment the delivery of oxygen, the hematopoietic growth factor erythropoietin is released, leading to an increase in red blood cells. ${ }^{16,17}$ Erythropoietin increases in the blood within hours of exposure to high altitude, but the actual increase in red cell mass in the peripheral blood requires 10 to 14 days. This relative polycythemia (eg, hematocrit $>58 \%$ ) minimizes the decrease in arterial oxygen content from the ambient hypoxic air. Polycythemia at very high levels, however, can impede oxygen delivery because of increased viscosity of blood. ${ }^{18}$ Here too, individual variability makes the advantage of erythropoiesis unpredictable, especially in athletes trying to gain some advantage in aerobic performance by training at altitude. ${ }^{19}$

The final step in the delivery of oxygen to the tissues is a function of blood flow (cardiac output) and arterial oxygen content. Cardiac output for any given level of exercise is the same at sea level and high altitude, but the process of getting oxygen from the blood to the cell membrane, cytosol, and mitochondria also relies on diffusion. Capillary and mitochondrial density over months at altitude may increase, resulting in more surface area for gas exchange at the cellular level, as well as decreasing diffusion distance for oxygen. The exact processes are an area of ongoing controversy, but in the setting of acute adaptations to altitude, there are mechanisms that require months to evolve that can improve peripheral oxygen delivery and utilization. ${ }^{7}$

\section{High-Altitude Illness and Injury Epidemiology}

High-altitude illnesses are a group of disorders that include acute mountain sickness (AMS), high-altitude cerebral edema (HACE), and HAPE. Thorough discussion about HAI is beyond the scope of this article, and excellent resources are available for those interested. ${ }^{20}$ These conditions comprise only a portion of the medical events at altitude.

The incidence of HAI varies across altitude sports and adventures worldwide. A review of Denali National Park Service (NPS) medical encounters between 1992 and
2011 among Denali (Mt. McKinley) mountaineers found that the most common complaints were altitude syndromes (29.6\%) and cold injuries $18.8 \%$ (18.1\% were frostbite), followed by pulmonary infections (8.4\%), musculoskeletal injuries (7.9\%), gastrointestinal/genitourinary complaints $(5.3 \%)$, and ENT infections $(4.6 \%){ }^{21}$ Other records from the Denali NPS found that falls accounted for $76 \%$ of all traumatic incidents resulting in search and rescue events. ${ }^{22}$ On Aconcagua, Argentina, Pesce et $\mathrm{al}^{23}$ in 2001 found a $39 \%$ prevalence of AMS among both trekkers and mountaineers. On Mt. Kilimanjaro, Tanzania in 2010, Meyer $^{24}$ examined a 6day ascent of the Machame route for trekkers, finding an overall incidence of AMS of $61 \%$, and $14 \%$ for HACE, and no cases of HAPE. Talbot et al in 2004 looked at a 10-day adventure race in Colorado starting at $9500 \mathrm{ft}$ $(2895 \mathrm{~m})$, with a maximum altitude of $13500 \mathrm{ft}(4100$ $\mathrm{m})$ and a cumulative elevation gain of $69400 \mathrm{ft}(21150$ $\mathrm{m})$, with a gain of $40000 \mathrm{ft}(12192 \mathrm{~m})$ in the first 12 hours. In this study, there was a $4.5 \%$ incidence of AMS at the start of the race and an overall incidence of $14.1 \%$, whereas $0.81 \%$ of participants were diagnosed with HAPE, leading to a dropout rate of $14 \% .^{25}$

\section{Risk Assessment for High-Altitude Illness}

Risk factors for altitude sports can be divided into those that are due to hypoxia and those due to other environmental factors such as increased ultraviolet radiation, cold, dehydration, etc. This article deals with the effects of altitude hypoxia, which may cause HAI, or aggravation of preexisting medical conditions. In addition, certain preexisting medical conditions can also predispose to HAI.

The main risk factor for HAI is the severity and rate of onset of hypoxic stress or the altitude reached and the rate of the ascent. Risk may be mitigated through acclimatization by allowing the body sufficient time to adjust to the hypoxia of altitude. The participant should provide a graph of the ascent profile to the physician, with the maximum altitude and sleeping altitude noted versus the days of the trip. This format allows easy recognition of potential risk. The physician and the participant can compare the ascent profile with the recommendations of the Wilderness Medical Society (WMS) guidelines and determine low, moderate, or high risk (Table 1, reprinted with permission ${ }^{26}$ ). For example, increasing sleeping altitude more than $500 \mathrm{~m}(1640 \mathrm{ft})$ per day when above $3000 \mathrm{~m}(9843 \mathrm{ft})$ is high risk, whereas days taken for acclimatization by staying at a given altitude for 2 nights will reduce risk. Abrupt ascent on initial entry into altitude is also a moderate to 
Table 1. Risk Categories for AMS (Reprinted With Permission ${ }^{26}$ )

\begin{tabular}{ll}
\hline Risk Category & \multicolumn{1}{c}{ Description } \\
\hline \multirow{2}{*}{ Low } & Individuals with no history of altitude illness and ascending to $\leq 2800 \mathrm{~m}$ \\
& Individuals taking $\geq 2$ days to arrive at $2500-3000 \mathrm{~m}$ with subsequent increases in sleeping elevation $<500$ \\
& $\mathrm{~m} / \mathrm{d}$ and an extra day for acclimatization every $1000 \mathrm{~m}$ \\
Moderate & Individuals with history of AMS and ascending to $2500-2800 \mathrm{~m}$ in $1 \mathrm{~d}$ \\
& No history of AMS and ascending to $>2800 \mathrm{~m}$ in $1 \mathrm{~d}$ \\
& All individuals ascending $>500 \mathrm{~m} / \mathrm{d}$ (increase in sleeping elevation) at altitudes above $3000 \mathrm{~m}$ but with an \\
& extra day for acclimatization every $1000 \mathrm{~m}$ \\
High & History of AMS and ascending to $\geq 2800 \mathrm{~m}$ in $1 \mathrm{~d}$ \\
& All individuals with a history of HAPE or HACE \\
& All individuals ascending to $>3500 \mathrm{~m}$ in $1 \mathrm{~d}$ \\
& All individuals ascending $>500 \mathrm{~m} / \mathrm{d}$ (increase in sleeping elevation) above $>3000 \mathrm{~m}$ without extra days \\
& for acclimatization \\
& Very rapid ascents (eg, $<7$-day ascents of Mt. Kilimanjaro)
\end{tabular}

Altitudes listed in the table refer to the altitude at which the person sleeps. Ascent is assumed to start from elevations $<1200 \mathrm{~m}$.

The risk categories described above pertain to unacclimatized individuals.

high risk, such as flying directly to $3000 \mathrm{~m}(9843 \mathrm{ft})$ or higher.

Regarding altitude entry and sleeping altitudes, it may be wise to spend the first night at a lower altitude, not often recommended in travel itineraries. For example, visitors trekking to Machu Picchu, Peru $(2400$ m/7972 $\mathrm{ft}$ ), will be better off spending the first night in the Urubamba Valley $(2870 \mathrm{~m} / 9420 \mathrm{ft})$ instead of Cusco $(3400 \mathrm{~m} / 11200 \mathrm{ft})$ after flying into Cusco.

The participant may be able to reduce risk of HAI of a high-altitude summit attempt if using a more conservative itinerary. Kilimanjaro (19 $341 \mathrm{ft} / 5895 \mathrm{~m}$ ), Tanzania, is commonly ascended quickly possibly due to a general lack of technical skills and equipment being required. For example, he/she may change an ascent to 8 or 9 days instead of 5 days, a common ascent profile. The figure represents a comparison of a typical guided ascent profile on Kilimanjaro, summiting in 5 days, which is considered high risk for HAI, versus a moderate-risk ascent in 8 days versus a low-risk ascent in 9 days if following recommendations by the WMS guidelines.

Medical history may also be useful, especially with a history of reproducible illness or freedom from illness with multiple ascents. A history of AMS after flying into La Paz, Bolivia (11 975 ft/3650 m), for a high-altitude peak in the Andes, for example, does not have the same significance as a history of severe AMS or HAPE at a ski resort in the Rocky Mountains [many of which range in altitude from over $2400 \mathrm{~m}(8000 \mathrm{ft})$ to nearly $3000 \mathrm{~m} \mathrm{(10}$ $000 \mathrm{ft}$ ) at base elevations]. Genetic factors may render some people particularly susceptible or resistant to HAI. Many individuals, however, being altitude naive, have no such history. In addition, rate of ascent and altitude reached always has to be taken into consideration. Often, an individual with a history of AMS with rapid ascent merely needs to go slower to prevent illness again.

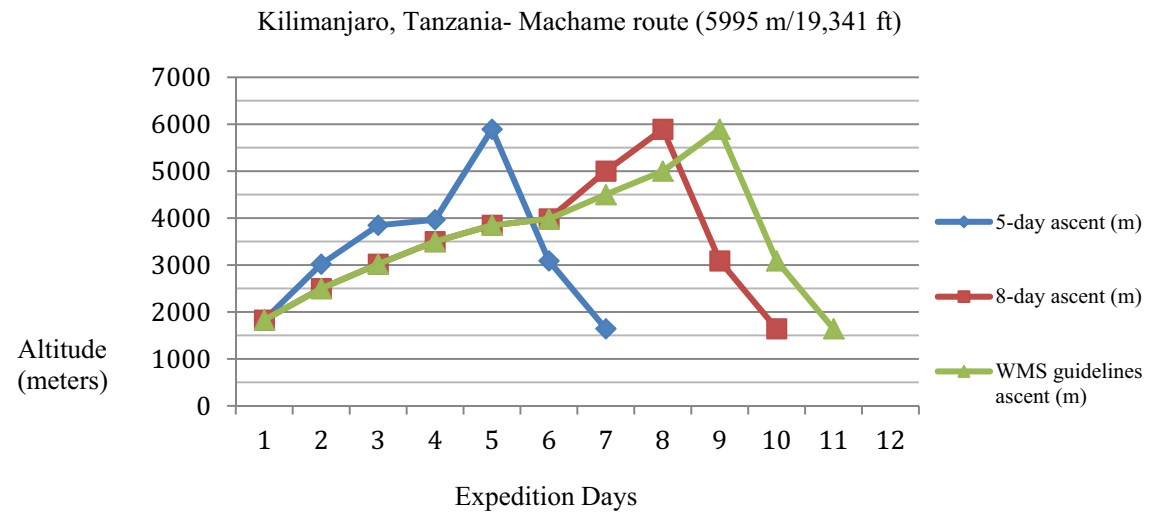

Figure. Comparison of different ascent profiles on a Kilimanjaro, Tanzania, showing a high-risk 5-day ascent versus moderate-risk 8-day ascent versus a low-risk schedule that follows the WMS Altitude Guidelines. 
A pitfall of the history is that it can be unreliable. The clinician needs to determine whether the previous illness was indeed because of the altitude. Individuals may report a prior episode of flu-like symptoms, for example, and attribute it to altitude illness.

Another element of the PPE for assessing risk of HAI is to elicit history, symptoms, or signs of a condition that might predispose to HAI. Any condition impacting oxygen transport may contribute to HAI. These may include poor ability to increase ventilation on ascent, such as carotid body dysfunction from surgery or radiation, neuromuscular disease affecting the thorax, kyphoscoliosis, and chronic obstructive pulmonary disease (COPD). Conditions impacting the transfer of oxygen from the alveolus to the blood include interstitial lung disease, COPD, pneumonia, and cystic fibrosis. Diminished arterial oxygen content from anemia can cause more breathlessness at altitude and diminished exercise performance but has not been linked to increased risk for HAI. Specific conditions causing increased risk for HAPE include any cause of pulmonary hypertension, congenital anomalies with shunts leading to increased pulmonary blood flow, or other abnormalities of the pulmonary circulation. Patent foramen ovale is associated with HAPE, but its importance is unclear. Conditions that might contribute specifically to risk of severe AMS or HACE include space-occupying lesions, idiopathic intracranial hypertension, and other causes of elevated intracranial pressure.

\section{Health Conditions Posing Risk Factors for High- Altitude Athletes/Adventurers}

A common reason for individuals to obtain a preparticipation consultation for traveling to high altitude is the presence of a chronic medical condition, even among athletes. There is a growing awareness that most medical conditions can be managed successfully in the hypoxic environment, and the medical literature on this topic is rapidly expanding. Clinicians need to be aware that recommending against high-altitude travel because of a medical condition, without investigating the topic, may

Table 2. Common Chronic Conditions Affected by Altitude

\begin{tabular}{ll}
\hline Lung Conditions & \multicolumn{1}{c}{ Cardiovascular Conditions } \\
\hline Asthma & ASHD \\
COPD & Hypertension \\
Sleep disordered & Cardiac arrhythmias, atrial fibrillation, \\
breathing, OSA, & premature ventricular contractions, \\
CSA & premature atrial contractions \\
& Congestive heart failure \\
\hline
\end{tabular}

no longer be acceptable to most participants. Common conditions specifically affected by or susceptible to altitude fall broadly into lung and cardiovascular conditions (Table 2). A number of recent reviews are available to assist the clinician with management of these conditions. ${ }^{27-29}$ In this article, the authors present a summary of the more common issues.

\section{LUNG DISEASE}

Chronic lung disease may be exacerbated at high altitude because of greater alveolar hypoxia and hypoxemia, increased pulmonary vascular resistance, and sleepdisordered breathing. The increase in ventilation may increase the work of breathing, although the decreased air density can improve airflow. Patients on oxygen at low altitude will need to increase $\mathrm{FiO}_{2}$ at altitude by the ratio of baseline barometric pressure/new barometric pressure. Monitoring arterial oxygen saturation with pulse oximetry provides guidance regarding the need for oxygen and titration to the desired value, which is generally around $90 \%$. The availability of inexpensive and convenient pulse oximeters makes this acceptable to participants. Supplemental oxygen is readily available in most resort areas, either with compressed gas cylinders or oxygen concentrators. Small portable oxygen concentrator devices are also available, are approved by the Federal Aviation Administration (FAA) for airplane use, and use both battery and AC power. Participants with mild COPD need to be in optimal and stable condition before ascent, supplied with medications to treat an exacerbation, have a pulse oximeter, be aware of local health care options, and have an evacuation plan for rapid transport to low altitude.

Individuals with asthma generally do better at high altitude because of reduced allergens and pollution, and high altitude is sometimes used as a treatment for asthma. However, exercise-induced bronchospasm (EIB) may be more problematic over $2500 \mathrm{~m}(8200$ $\mathrm{ft}$ ), because of ventilation of large volumes of cold dry air and resultant respiratory heat loss. Durand et $\mathrm{al}^{30}$ found that $50 \%$ of highly trained ski mountaineers in the Alps developed EIB during racing, yet only one fourth of this group had been previously diagnosed by a physician with EIB. Risk indicators for asthma exacerbation at high altitude include the use of inhaled bronchodilators more than 3 times a week before travel and intensive physical exertion at high altitude. ${ }^{4}$ Other important recommendations for asthmatics ascending to high altitude include medical optimization of function before ascent, continuation of baseline asthma medications, and having access to corticosteroids and bronchodilators in the event of exacerbation. ${ }^{4}$ The use of an airway 
warming mask may be helpful, but its benefit has not been proven. There is no evidence that asthma may predispose to HAPE. If the examiner is not comfortable with this assessment, then participants with pulmonary vascular disorders should be referred to a pulmonologist or cardiologist with an interest in high altitude or to a high-altitude specialist for consultation.

Sleep-disordered breathing is a common reason for a PPE before altitude exposure. In general, obstructive sleep apnea (OSA) improves at high altitude, apparently because of increased upper airway muscle tone. However, central sleep apnea (CSA) develops in those with OSA or is exacerbated at high altitude. For this reason, acetazolamide should be considered for all participants with OSA or CSA traveling to high altitude. Latshang et $\mathrm{al}^{31}$ have shown marked improvement in the apneahypoxia index and oxygen saturation with the use of acetazolamide, with or without the use of continuous positive airway pressure (CPAP). Participants with moderate to severe OSA using CPAP should take their machines with them to altitude if possible. Newer battery-powered devices are available but require frequent charging. Currently available CPAP devices have automatic pressure compensation for altitude and do not need to be adjusted.

\section{CARDIOVASCULAR CONDITIONS}

Healthy persons have a slight increase in blood pressure on ascent to high altitude. Individuals with hypertension tend to have a quite variable response to hypoxia, and although the increase can be 30 to $40 \mathrm{~mm} \mathrm{Hg}$ systolic pressure, this response is transient and resolves with descent. Such changes are unusual below $3000 \mathrm{~m}(9840 \mathrm{ft})$ and more common as altitude increases above $3000 \mathrm{~m}(9840 \mathrm{ft})$. Because these hypertensive responses are transient and not clinically significant, participants should not be advised to monitor their blood pressure at altitude unless it is labile at low altitude. ${ }^{32}$ Participants with hypertension need to continue their medications at altitude. Should a participant become symptomatic from hypertension at altitude and require treatment, the ideal agent has not been established. The best approach may be to increase the individual's current medication and add extra medications only if absolutely necessary. For participants with labile hypertension or resistant hypertension at low altitude, the use of nocturnal oxygen at high altitude may help maintain blood pressure control, especially in the elderly.

Individuals with arteriosclerotic heart disease (ASHD) tend to self-select participation in wilderness and altitude adventures, and only those with very stable disease usually undertake such activities. These individuals generally do well. The available data suggest that persons who are accustomed to regular exercise regardless of their underlying disease will do well at moderate altitude, up to $3000 \mathrm{~m}$ (9840 ft). People who have had revascularization procedures and a normal exercise test have traveled above $5500 \mathrm{~m}(18045 \mathrm{ft})$ without difficulty. Risk stratification should include history of current symptoms, recent ischemic events, medical history, and recent results of exercise treadmill, stress echo, or other exercise imaging techniques. For the participant with no known ASHD but with risk factors, traditional risk stratification is appropriate. Again, emphasis should be on fitness in these individuals, with the ability to perform physical exercise at home congruent with the planned activity at altitude. Data from Austria indicated that the only increased risk of sudden cardiac death at altitude was in men who were sedentary at low altitude. ${ }^{33}$

The increase in catecholamines on ascent to altitude could provoke or aggravate a cardiac arrhythmia. Premature ventricular contractions are more common at high altitude, but whether dangerous arrhythmias may be more common is unclear. No systematic studies are available to help advise clinicians on this issue. Individuals with poorly controlled and possibly dangerous arrhythmias should be advised not to travel to high altitude. Participants with stable atrial fibrillation, paroxysmal atrial fibrillation, or other atrial tachycardia should be advised to have rescue medication with them, such as a beta blocker, in case of exacerbation. Benign premature atrial contractions do not pose risk at high altitude.

Individuals with congestive heart failure need to be informed about possible adverse effects of high altitude. Medications such as furosemide need to be maintained and may be increased if weight gain or edema develops. Patients with congestive heart failure should take careful precautions to avoid AMS, with its attendant fluid retention. This disease needs to be well compensated, as well as having established cardiovascular fitness before travel.

\section{Prevention of High-Altitude Illness}

The general principles for prevention of HAI include reducing hypoxic stress through a reasonable rate of ascent and low sleeping altitude, the use of chemoprophylaxis as necessary, and management of preexisting medical conditions that might contribute to HAI. Specific management depends on risk assessment, as above. A slower rate of ascent may reduce risk from high to low or medium and obviate the need for prophylaxis. Individuals with a history of altitude illness with rapid ascent may merely need to ascend more slowly. According to the 
WMS guidelines, individuals with low risk do not require chemoprophylaxis, individuals with moderate risk may be offered chemoprophylaxis, and those with high risk should be encouraged to use chemoprophylaxis. ${ }^{26}$

These WMS guidelines go on to make specific recommendations for medication use. Individuals susceptible to AMS or at high risk are advised to consider acetazolamide, the drug of choice for HAI prevention, at an oral dose of $125 \mathrm{mg}$ every 12 hours. The medication is continued until a maximum altitude has been achieved or acclimatization has occurred. Acetazolamide is also useful for treating nocturnal periodic breathing of high altitude. Treatment for AMS is initiated at $250 \mathrm{mg}$ every 12 hours, with or without accompanying descent to a lower altitude. Mild symptoms may be monitored at the current altitude. For severe symptoms or clinical HACE, oral dexamethasone $4 \mathrm{mg}$ every 6 hours should be initiated. For individuals susceptible to HAPE, nifedipine $30 \mathrm{mg}$ (long acting) every 12 hours is used for prophylaxis. For treatment of clinical HAPE, oxygen if available is the ideal medication, and if not available, nifedipine $30 \mathrm{mg}$ (long acting) every 12 hours should be administered, while preparations are made for descent. ${ }^{26}$

The participant should also be advised to avoid respiratory depressants such as alcohol and benzodiazepines, pay attention to hydration, avoid overexertion by slowing one's pace or intensity of exercise compared with low altitude, and to rest more. Supplemental oxygen is the ideal agent for preventing HAI in those particularly susceptible or who prefer oxygen to medication.

Preacclimatization may be a useful strategy. Multiple studies confirm that exposure to either actual or simulated altitude before a sojourn to altitude can reduce AMS and improve exercise performance. Schneider et $\mathrm{al}^{34}$ showed that only 2 nights or more at $3000 \mathrm{~m}$ (9840 ft) in the 8 weeks before an ascent to $4559 \mathrm{~m} \mathrm{(14}$ $960 \mathrm{ft}$ ) reduced AMS, and the more nights the greater the protection. Suggesting that clients spend a few days and nights at $2500 \mathrm{~m}(8200 \mathrm{ft})$ or higher can be very helpful for medium-altitude treks and athletic events, such as 2500 to $3000 \mathrm{~m}$ (8200-9840 ft). Preacclimatization periods of a week or more can be useful for higher altitude sojourns. Hypobaric hypoxia is more effective than normo-baric hypoxia, for reasons that are unclear. With normobaric hypoxia, a minimum time of 8 hours per day for at least 7 days is necessary to effectively reduce AMS and improve performance. Realistically, this means sleeping in a hypoxic tent or chamber. Although brief hypoxic exposures with the use of mask, chamber, or a nitrogen-filled room, for minutes to a few hours at a time, are being touted as a means to preacclimatize, the data do not support these brief techniques for preventing AMS. Whether they may improve performance at sea level or high altitude is also unclear.

\section{Approach To A High-Altitude Sports/ Adventure Preparticipation Evaluation}

Terminology that is standardized is difficult to achieve. This is in part because this article refers to high-altitude adventures in terms of sports or other expeditions. Although some are private events, others are organized or guided. For this reason, the authors suggest an expanded title to include this spectrum.

Basic principles of PPE can be applied to those involved in high-altitude sojourns. Identify conditions placing an individual at risk for illness or injury (include personal and family history of HAI) recognize potentially life-threatening conditions preventing safe participation and optimize health conditions to promote success in high-altitude activities. For the high-altitude athlete/adventurer, the history section of the PPE should focus on the specifics of the ascent, the presence of pulmonary and cardiovascular conditions, a typical review of systems to identify any other acute or chronic conditions, and medications/supplements that could potentially contribute to altitude illness. Alcohol and caffeine withdrawal need to be considered in individuals with habitual use. Conducting a physical examination is recommended when possible, but off-site consultation may not afford this opportunity. The physical examination (if performed) should focus on the cardiac and pulmonary systems. Table 3 presents an example of a health history form that could be completed by participants before an evaluation to guide the clinician during the PPE.

\section{Other Considerations for Travel To High Altitude}

Although history alone detects the majority of medical and musculoskeletal conditions, ${ }^{5}$ the physical examination should include vital signs, visual acuity, pulmonary, and cardiovascular examinations and focus on any active or chronic musculoskeletal conditions that may impact a participant's ability to safely ascend or be easily managed before a trip or expedition. If not trained in performing a sports-specific evaluation (essential to understand activity-specific epidemiology), then the authors recommend referring the PPE to a trained clinician. A more thorough evaluation, potentially including laboratory testing or imaging studies, will be recommended if deemed necessary by a trained clinician with prudence to avoid overscreening and redundancy.

Participants should also be counseled on risks of carbon monoxide poisoning during camps, wound care, sanitation, and avoidance of frostbite. Additional 
Table 3. Health History Form for High-Altitude Trekking, Climbing, and Other Activities

Part I: Individual and Expedition Information

Name:

DOB/Age:

Gender:

Planned expedition (location, peak or trek):

Summit/highest planned altitude:

Date of planned departure:

Total trip days allowed:

Part II: Altitude experience and history of altitude illness

Complete each response below to the best of your ability. List the elevation if known.

Provider Notes

(Administrative use only)

What is the highest altitude you have reached?

How many days did you spend getting to that altitude?

What is the highest altitude you have slept?

How many nights did you sleep at that altitude?

Possible symptoms of altitude illness

Yes

No

Elevation

Provider Notes

(Administrative use only)

Headache

Nausea

Vomiting

Lightheadedness

Difficulty sleeping

Shortness of breath

Cough

Exercise intolerance

Problems walking or standing

Confusion

Have you ever been diagnosed with any of the following conditions? If so, list elevation.

Yes No Elevation Provider Notes

(Administrative use only)

Acute mountain sickness (AMS)

High-altitude pulmonary edema (HAPE)

High-altitude cerebral edema (HACE)

Part III: Personal history of chronic cardiovascular and pulmonary conditions posing risk at altitude

Cardiovascular conditions

Yes

No

Date of Diagnosis

High blood pressure or taking medication for blood

pressure

Previous heart attack or heart disease (history of

angioplasty, stenting, CABG)

Heart arrhythmia

Congestive heart failure

Lung conditions

Asthma

COPD/Emphysema

Sleep apnea (obstructive or central)

Use of a CPAP machine

Part IV: History of other acute medical conditions occurring at altitude

Check any condition you have experienced at altitude in the past. List elevation if known.

$\begin{array}{llll} & \text { Yes } & \text { Novation if known }\end{array}$

Carbon Monoxide

Yes $\quad \mathrm{N}$

Frostbite

Gastrointestinal

Musculoskeletal

Respiratory

Skin condition or wound issue

Vision (sun or snow related, blindness, other) 
counseling should involve issues of personal safety during climbing to avoid falls and environmental exposures such as wind, sun, and other climate specific factors. Appropriate fitness and activity-specific conditioning should be advised, and stability of wellcontrolled chronic conditions should be established. Planning for medical kits that include first aid materials and adequate supplies of chronic medications or medications for specific issues should also be discussed. Clinicians may also suggest participants look into options for travel and rescue insurance.

\section{Summary}

At high altitude, inherent risks exist from both environmental factors and personal health conditions. As with traditional or organized sports, wilderness sports, activities, and other adventures will benefit from a formal PPE to include the same basic principles. This will include the same basic principles as outlined in PPE Preparticipation Physical Evaluation, fourth edition. ${ }^{5}$ In addition to these fundamental principles, knowledge of altitude physiology, altitude injury, and illness epidemiology will help guide the high-altitude activities/adventures PPE. Understanding how chronic cardiovascular and lung conditions pose risk at altitude is also essential. By reviewing the health history of an individual going to altitude for sport or adventure, a clinician can risk stratify and appropriately counsel participants about prevention and treatment of altitude conditions, monitoring of chronic conditions, use of medications, and proper training. Diagnostic studies and/or performance testing should be used when indicated but is not universally recommended. Medical kits should include supplies specific to the environment, personal medications for chronic conditions or acute issues, and prophylactic medications for known high-altitude susceptible conditions. Additional issues such as $\mathrm{CO}$ poisoning, wound care and infection control, as well as frostbite prevention and management and considerations for travel/rescue insurance should also be addressed. With careful PPEs by trained clinicians as well as personal planning, altitude-seeking athletes can increase their chances for a successful adventure.

\section{References}

1. Davies A, et al. Determinants of summiting success and acute mountain sickness on Mt Kilimanjaro (5895 m). Wilderness Environ Med. 2009;20:311-317.

2. Sagamartha National Park. Sagamartha National Park, Jorsale Entrance Station Records. Solu Khumbu, Nepal: Sagamartha National Park; 2015.
3. Hackett PH, Roach RD. High altitude medicine and physiology. In: Auerbach PS, ed. Wilderness Medicine. Philadelphia, PA: Elsevier; 2012:2-32.

4. Doan D, Luks AM. Wilderness and adventure travel with underlying asthma. Wilderness Environ Med. 2014;25: 231-240.

5. Bernhardt DT, Roberts WO. PPE Preparticipation Physical Evaluation. 4th ed. Elk Grove Village, IL: American Academy of Pediatrics; 2010.

6. Roi G, Giacometti M, von Duvilliar S. Marathons in altitude. Med Sci Sports Exerc. 1999;31:723-728.

7. Schoene RB. Training for the wilderness adventure. In: Auerback PS, ed. Wilderness Medicine. Philadelphia, PA: Elsevier Mosby; 2012:1928-1936.

8. Fulco C, Rock P, Cymerman A. Maximal and submaximal exercise performance at altitude. Aviat Space Environ Med. 1998;69:793-801.

9. Sutton J, et al. Operation Everest II: oxygen transport during exercise at extreme simulated altitude. $J$ Appl Physiol (1985). 1988;64:1309-1321.

10. Wehrlin J, Hallen J. Linear decrease in VO2max and performance with increasing altitude in endurance athletes. Eur J Appl Physiol. 2006;96:404-412.

11. West J, et al. Pulmonary gas exchange on the summit of Mount Everest. J Appl Physiol Respir Environ Exerc Physiol. 1983;55:678-687.

12. Schoene R. Limits of human lung function at high altitude. J Exp Biol. 2001;204:3121-3127.

13. Schoene R, et al. Relationship ofhypoxic ventilatory response to exercise performance on Mount Everest. J Appl Physiol Respir Environ Exerc Physiol. 1984;56: 1478-1483.

14. Gale $\mathrm{G}$, et al. Ventilation-perfusion inequality in normal humans during exercise at sea level and simulated altitude. JAppl Physiol (1985). 1985;58:978-988.

15. Wagner P, Mitzner W. Effect ofhypoxia on bronchial circulation. J Appl Physiol (1985). 1988;65:1627-1633.

16. Semenza G. Involvement ofoxygen-sensing pathways in physiologic and pathologic erythropoiesis. Blood. 2009;114:2015-2019.

17. Winslow N, Samaja M, West J. Red cell function at extreme altitude on Mount Everest. J Appl Physiol Respir Environ Exerc Physiol. 1984;56:109-116.

18. Winslow R, et al. Effects of hemodilution on $\mathrm{O} 2$ transport in high-altitude polycythemia. J Appl Physiol (1985). 1985;59:1495-1502.

19. Chapman R, Stray-Gundersen J, Levine B. Individual variation in response to altitude training. J Appl Physiol (1985). 1998;85:1448-1456.

20. Auerbach P. Wilderness Medicine. 6th ed. Philadelphia, PA: Elsevier Mosby; 2012:2277.

21. McIntosh S, et al. Mountaineering medical events and trauma on Denali, 1992-2011. High Alt Med Biol. 2012;13:275-280.

22. McIntosh S, et al. Search and rescue activity on Denali, 1990 to 2008. Wilderness Environ Med. 2010;21: 103-108. 
23. Pesce $\mathrm{C}$, et al. Determinants of acute mountain sickness and success on Mount Aconcagua (6962 m). High Alt Med Biol. 2005;6:158-166.

24. Meyer J. Twice-daily assessment of trekkers on Kilimanjaro's Machame route to evaluate the incidence and timecourse of acute mountain sickness. High Alt Med Biol. 2012;13:281-284.

25. Talbot T, Townes D, Wedmore I. To air is human: altitude illness during an expedition length adventure race. Wilderness Environ Med. 2004;15:90-94.

26. Luks AM, et al. Wilderness Medical Society practice guidelines for the prevention and treatment of acute altitude illness: 2014 update. Wilderness Environ Med. 2014;25(4 Suppl): S4-S14.

27. Luks A, Swenson E. Travel to high altitude with pre-existing lung disease. Eur Respir J. 2007;29: 770-792.

28. Hoigne P, Gibbs J. Can my patient with CVD travel to high altitude? Practitioner. 2013;257:27-30.
29. Luks AM, Hackett PH. High altitude and common medical conditions. In: Swenson ER, Bartsch P, eds. Human Adaptation to Hypoxia. NY: Springer; 2014:449-477.

30. Durand F, et al. Undiagnosed exercise-induced bronchoconstriction in ski-mountaineers. Int $J$ Sports Med. 2005;26:233-237.

31. Latshang $\mathrm{T}$, et al. Effect of acetazolamide and autoCPAP therapy on breathing disturbances among patients with obstructive sleep apnea syndrome who travel to altitude: a randomized controlled trial. JAMA. 2012;308:2390-2398.

32. Luks AM. Should travelers with hypertension adjust their medications when traveling to high altitude? High Alt Med Biol. 2009;10:11-15.

33. Burtscher M, Ponchia A. The risk of cardiovascular events during leisure time activities at altitude. Prog Cardiovasc Dis. 2010;52:507-511.

34. Schneider M, et al. Acute mountain sickness: influence of susceptibility, preexposure, and ascent rate. Med Sci Sports Exerc. 2002;34:1886-1891. 